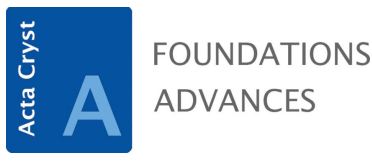

ISSN 2053-2733

\section{The Physics of Solids. By J. B. Ketterson. Oxford University Press, 2016. Pp. 1056. Price GBP 55.00 (hard- back). ISBN 9780198742906.}

\author{
Ernesto Estevez-Rams*
}

Facultad de Fisica, Universidad de la Habana, Cuba. *Correspondence e-mail: estevez@fisica.uh.cu

There is a prestigious tradition of textbooks on solid-state physics starting from the early days of quantum mechanics. Solid state has been the playground where essential tenets of quantum mechanics are put to the test. There are at least two ways in which solid-state physics has been approached didactically. In a first approach, theory is built from the ground and the basic phenomena are then seen as a consequence of such ' $a b$ initio' construction. The emphasis here is in being as rigorous as quantum models and mathematical tractability allow. A second approach takes the reader on a tour of as many areas of solid-state physics as the length of the book allows, while depending heavily on phenomenology. Here simplicity is sought, but not to the extent that the exposition of the subject becomes useless. The intention of the author is to give something more than a bird's eye view of solid-state physics without describing every detail of the extremely complex landscape. The book from Ketterson belongs to the second approach.

A lot has changed since Kittel's classical book on solid-state physics and, at the same time, a lot remains the same. After all, quantum mechanics and electrodynamics have not changed since then, but better characterization techniques, new materials, and the increasing exploration of low dimensionality and high energies have brought to light phenomena previously hard to observe or not seen at all in yesterday's studies. Ketterson's book is welcomed as a valuable and fairly successful attempt to expose solid-state physics from today's perspective. This is a thick book of over 1000 pages long, not the kind you carry with you while travelling, even for a scientist. But if one sees the amount of material it covers in 49 chapters, even as thick as it is, it can but only skim each subject. The challenge is to make it understandable and useful to the reader.

The book starts by introducing fundamental notions in elasticity theory, electrodynamics, thermodynamics and physical statistics, focused on their application to solidstate media. Here, concepts such as stress and strain, heat capacity, Fermi gas and electrons in solids, as well as elemental statistical models of magnetism are introduced. The basic and fundamental models for each phenomenon are described. A chapter on crystal bonding is also incorporated in this introductory material. It assumes that the reader has some knowledge of classical and quantum mechanics as well as electrodynamics, at least to the level of an undergraduate semester. The introductory topics are followed by a two-chapter part on crystal structure and X-ray diffraction. This is followed by electronic band theory in periodic crystals, where the ubiquitous Bloch theorem and functions are introduced together with the usual models of free electron gas, orthogonalized plane wave, pseudo-potential, binding, augmented plane waves and Green function methods. In Part IV, electron-electron interaction is studied under HartreeFock and density functional theory. Part $\mathrm{V}$ is about phonons and lattice dynamics, including heat transport and thermal expansion. Having treated both electrons and phonons in solids, Part VI returns to transport properties of electrons in solids, including the treatment of scattering at static centres as well as with phonons. With most of the fundamental models for both static systems and transport being covered, Parts VII, VIII, IX and XI focus on specific types of materials and their properties: semiconductors, insulators, magnetic materials and superconductors, respectively. In Part X, optical properties are studied. The author makes an unjustified detour in Part XI by treating superfluidity in helium three and four, together with Landau theory, which are not really subjects in solid-state physics, which gives the impression that these chapters belong to another book. Part XII is about disordered materials, a subject not usually covered in 
books on solid-state physics and therefore welcomed. The main types of defects are introduced, including grain boundaries, and transport properties in the presence of disorder are explained. In this part magnetic impurities and the corresponding Anderson model and Kondo effect are also looked at. Finally, Part XIII, named 'Special topics', covers strongly correlated systems, high-temperature superconductors, patterned materials and low-dimensional artificial solids as well as the quantum Hall effect, ending with a chapter on carbon-based nanostructures such as fullerenes, nanotubes and graphene.

When one starts reading Ketterson's book one wonders why it did not start with a basic, yet thorough, treatment of crystallography. Crystallography is not treated until Part II and yet, in the introductory material of Part I, constant reference is made to crystallographic notions, without which much of the topics will remain too general. This is particularly the case while treating elastic properties of solids in Chapter 1, where reference to cubic and hexagonal materials is made and there is a section on elastic moduli in those crystal systems (1.5). The same goes for crystal bonding in Chapter 7, where covalent bonding is studied in the cubic diamond structure and in graphite. This reference to topics further on, in order to explain current treatments, is on occasion unavoidable, but that does not seem to be the case here.

A more serious concern arises while reading Part II, where the bulk of crystal structure is explained. Crystallography is treated in a very simple way, perhaps too simple. For example, the distinction between primitive cell and unit cell is not made, which leads to the definition of primitive lattice basis vectors over unit cells without regard to whether they are primitive or not. The definition of isometries is not really ever given. Rotations and reflections are introduced straightforwardly, but there is hardly any mention of the inversion operation, while glide and screw rotations are just mentioned in brief. A reader not acquainted with any basic crystallographic knowledge will find it hard to understand the stereographic representation of the 32 types of crystallographic point group, nor will she or he understand what symbols like $\overline{3} m$ actually mean. There is no serious attempt to explain the international notation. I am well aware that this simplistic approach to crystallography follows an unfortunate tradition of many books in solid-state physics, and in this regard the book performs no better or worse than others, but this cannot be used as a justification. Crystallography is not another topic in solid-state science; it is one of the fundamental, if not the fundamental, building block on which much of the theory is built. Mistakes or oversimplifications and, at the same time, obscure treatment of basic topics in crystal structure description can make it difficult for the reader to follow afterwards the rationale behind the construction of physical models describing properties and behaviours of solids. For example, the construction and definition of the Wigner-Seitz cell are treated poorly, as the author wrongly identifies lattice points with atoms in the face-centred cubic (f.c.c.) and body-centred cubic (b.c.c.) structures. The Wigner-Seitz construction is not properly described, as it is introduced through two examples with the hope that this will suffice for the reader to understand what is going on. It does not. Reciprocal-space treatment is another subject treated poorly. Apart from using a nonstandard notation of $m 1, m 2, m 3$ instead of the usual $h, k, l$ symbols, a factor of $2 \pi$ in the definition of the reciprocal vectors is an unfortunate choice, which leads to having the length of the reciprocal vectors equal to $2 \pi$ times the inverse of the interplanar distance. There seems no need for this additional $2 \pi$ in all these relations and, certainly, this is not a commonly used convention in crystallography. When it comes to X-ray diffraction, there is no improvement over the too simplistic approach already seen in the crystallography chapter. I can hardly imagine that a reader will come to understand, from reading the two short paragraphs that the author devotes to it, the Ewald construction and its importance, which is impossible to overstate, for this subject. It would appear that the Ewald construction, if understood at all from the obscure description, is a mere curiosity not used in what follows and the reader may wonder what it is useful for.

Surprisingly, the structure factor is explained before atomic scattering. The whole treatment is too sketchy which makes it hard to follow for anyone new to the subject. Reflection conditions are hardly mentioned, with a single reference to the b.c.c. lattice. I find it unlikely that the reader will be able to gain any useful information from this. 'Experimental methods' mentions only the rotating crystal, powder and the Laue method. No mention is made of the phase problem and how the complete structure of a crystal can be solved. In a book where current topics are discussed, there is no mention of synchrotron sources, neutron diffraction or any new development in crystal studies. I miss a simple note on quasicrystals and incommensurate structures. Since disorder is treated further on in the book, I believe this would be the place to at least introduce defects in crystal solids and their effect on diffraction.

To summarize, regarding the treatment of crystallography, the reader will be better off getting their crystallography background somewhere else and returning to the book for other subjects which are better treated.

The strength and weakness of Ketterson's book lie in the vast amount of topics it touches. A careful balance must be made between the length of the book and how deep one goes into a specific subject. A line must be drawn so as not to cut so much from a subject as to make it hardly informative. In general, the author saves the book from crossing that line in most chapters and at the same time covers enough material in a single book to make it a wealth of information. The chapter on magnetism is a good example. Necessarily brief, yet it succeeds in being informative, covering basic topics like the Weiss phenomenological approach to magnetic materials and Landau thermodynamical treatment of magnetic phase transition and more advanced ones like magnetic anisotropy, domains and domain walls. This is followed by a chapter on dynamical properties of magnetic materials, which then covers additional topics such as magnetization reversal, spin waves, magnons and even some basic ideas in micromagnetism. This is further followed by a chapter on resonance. This way of 
treating a subject, when it comes to discussion of physical properties and behaviour, is the strength of the book. I could go on referring to other topics in the same manner.

One thing I missed was a chapter dealing briefly with amorphous materials; this is seldom treated in solid-state physics books. A lot of wonderful insight has been gained in the last decades into these types of solids that I think certainly deserves to be included in solid-state books. One could argue that the book is lengthy enough without adding a further subject, but I believe that amorphous solids are better justified in a solid-state book such as this, than the more than 50 pages devoted to superfluidity.

The exercises at the end of each chapter are not the strongest area of the book; however, they are well placed and cover the topics being explained.
When reviewing a book of more than 1000 pages, it is impossible to refer to every subject it touches. One must take into consideration that this type of book serves different purposes. It can be used as a textbook, in which case it would probably need to be accompanied by other books to fill some gaps. But this can be said of almost all solid-state physics books with a mixed phenomenological-theoretical approach. At the same time, as a textbook, it offers enough material for a two-semester course, and will give students a broad and good enough insight into the modern realm of solid-state physics. It can also be used as a reference book; the kind of reading material you return to over the years, when you are suddenly seeking an accessible answer to a basic question that pops up in front of you by some Murphy's law accident. In this respect, this book certainly hits the mark. 